\title{
Die Vermittlung der Ungleichheit: Zur symbolischen Ordnung sozialer Unterschiede und wohlfahrtsstaatlicher Rationalität in narrativen Konstruktionen der Mittelschicht
}

\author{
Marlon Barbehön
}

Angenommen: 29. Juni 2021 / Online publiziert: 20. Juli 2021

(C) Der/die Autor(en) 2021

Zusammenfassung Die Frage, wann eine Gesellschaft soziale Differenzen als Ungleichheiten erfährt und zum Gegenstand öffentlicher Kontroversen erhebt, lässt sich nicht anhand eines objektiven Maßstabs beantworten. Ungleichheit verweist auf kontingente Deutungs- und Interpretationsleistungen, in denen beobachtbare Differenzen nicht nur als Unterschiede behandelt, sondern in Ungleichheiten transformiert werden. Dabei kommt dem Modus der Erzählung eine wesentliche Rolle zu, da er es möglich macht, gesellschaftliche Komplexität auf ein erfassbares Maß zu reduzieren und ambivalente Phänomene zumindest temporär auf eine bestimmte Bedeutung festzulegen. Der vorliegende Beitrag erörtert die Logik und Funktionsweise derartiger erzählerischer Transformationsleistungen anhand der Kategorie der Mittelschicht, die aufgrund ihrer symbolischen Logik bei narrativen Verarbeitungen sozialer Verhältnisse eine besondere Performativität entfaltet. Anhand der bundesdeutschen medialen Öffentlichkeit wird rekonstruiert, in welche Erzählungen „die Mittelschicht" eingebunden ist und wie hierüber eine kollektiv wirksame symbolische Ordnung von Ungleichheit sowie korrespondierende Vorstellungen über ,rationales“ wohlfahrtsstaatliches Regieren etabliert werden. Es werden vier Narrative unterschieden - das Metanarrativ der Mittelschichtsgesellschaft sowie die Narrative der verdienstvollen, der bedrohten und der bevorzugten Mittelschicht -, die ein je eigenes Bild von der Gestalt, den Ursachen, den Auswirkungen und der (Il-)Legitimität von Ungleichheit zeichnen und in ihrer Gesamtheit ein kollektives Wissen über soziale Unterschiede und deren Regierbarkeit etablieren. Der Beitrag entschlüsselt somit die narrative Vermittlung von Ungleichheit in zweierlei Weise: zum einen als Praxis der Übermittlung von gesellschaftlicher Komplexität in die Sphäre kollektiver Wahrnehmbarkeit und zum anderen als Praxis der Bezugnahme auf Vorstellungen

\footnotetext{
Marlon Barbehön ( $\square)$

Institut für Politische Wissenschaft, Universität Heidelberg, Bergheimer Straße 58, 69115 Heidelberg,

Deutschland

E-Mail: marlon.barbehoen@ipw.uni-heidelberg.de
} 
von der gesellschaftlichen Mitte, die als beweglicher Referenzpunkt fungiert, um Ungleichheit von ,bloßen“ Differenzen zu unterscheiden.

Schlüsselwörter Soziale Ungleichheit · Mittelschicht · Wohlfahrtsstaat ·

Narrativanalyse $\cdot$ Diskursanalyse $\cdot$ Interpretative Policy-Analyse

\title{
Centring inequality: On the symbolic order of social differences and welfare state rationality in narrative constructions of the middle class
}

\begin{abstract}
The question when a society observes and debates social differences as inequalities could not be answered on the basis of an objective indicator. Inequality is contingent upon practices of interpretation and evaluation in which observable differences are not only treated as differences but transformed into inequalities. Narratives are of crucial importance here as they enable to reduce social complexity and to ascribe a certain meaning to ambivalent social phenomena. This contribution investigates the logic of these narrative transformations by focussing on the category of the middle class which, due to its symbolic architecture, features a specific narrative performativity. By analysing German media coverage, the contribution reconstructs the narratives in which "the middle class" is entangled and how these narratives establish a symbolic order of inequality and accompanying ideas about "rational" ways of welfare state governing. Four narratives are distinguished-the metanarrative of the middle-class society and the narratives of the meritorious, the threatened and the privileged middle class_-, each drawing a specific picture about the shape, causes, effects and (il-)legitimacy of inequality. In sum, these narratives establish a collective knowledge about inequality and how it could be governed. The contribution thus decodes the narrative centring of inequality in two ways: first, as a practice of mediation in which social complexity is conveyed into the sphere of collective perceptibility, and second, as a practice of linkage in which observable differences are related to imaginations of the middle class as a moving reference point for discerning inequality from "mere" differences.
\end{abstract}

Keywords Social inequality $\cdot$ Middle class $\cdot$ Welfare state $\cdot$ Narrative analysis · Discourse analysis $\cdot$ Interpretive policy analysis

\section{Einleitung}

Die Existenz sozioökonomischer Differenzen gehört zu den Wesensmerkmalen moderner, marktwirtschaftlich organisierter Gesellschaften. Obgleich weder natur- noch gottgegeben, sind wertvolle Güter, und mit ihnen individuelle Lebenschancen, immer zu einem gewissen Grade ungleich verteilt; auch in den egalitärsten Gesellschaften unserer Welt haben einige (wenige) mehr als (viele) andere. Inwiefern derartige Unterschiede jedoch als Ungleichheit erfahren werden, lässt sich nicht anhand einer „objektiven Messung“ (was immer damit gemeint sein mag) bestimmen. Jenseits der methodischen Schwierigkeiten, die Komplexität von Ungleichheit über Zahlen abzubilden, ist die Transformation von beobachtbaren sozioökonomischen 
Differenzen in soziale Ungleichheit an einen normativen Maßstab gebunden, der darüber entscheidet, bis zu welchem Punkt Unterschiede als ,normal“, ,,vernachlässigbar“, „,unausweichlich“ oder ,gerechtfertigt“ gelten und ab wann sie ,problematisch“, „dysfunktional“, „unangemessen“ oder ,skandalös“ erscheinen. Dieser Maßstab wiederum ist nicht naturgegeben, sondern gebunden an gesellschaftliche Werturteile und kollektive Deutungsleistungen. Soziale Ungleichheit ist, wie Nicole Burzan (2011, S. 7) gleich zu Beginn ihres Standardwerks unmissverständlich festhält, ,eine gesellschaftliche Konstruktion, die an ihre historische Zeit gebunden ist und nie ,objektiv" sein kann“.

Die soziohistorische Kontingenz von Ungleichheit verweist mithin auf Praktiken der kollektiven Sinnstiftung, in denen fortdauernd verhandelt wird, wann Unterschiedlichkeit in Ungleichheit umschlägt, wann also Differenz als Gefährdung des für moderne demokratische Gesellschaften konstitutiven Prinzips der Gleichheit zu werten ist. Im Falle der bundesdeutschen Gesellschaft (vermutlich aber auch weit über sie hinaus) offenbart ein flüchtiger Blick sowohl in die politisch-mediale Öffentlichkeit als auch in den sozialwissenschaftlichen Diskurs, dass Aushandlungen dieser Art zumeist unter binärem Vorzeichen verlaufen, etwa wenn eine Spaltung der Gesellschaft in „oben“ und ,unten“ oder eine „Schere zwischen Arm und Reich“ diagnostiziert wird. In diesen Bildern schwingt die Vorstellung eines Zwischenraums mit - die gesellschaftliche Mitte -, der als archimedischer Punkt fungiert: Differenzen werden insbesondere dann als Problem erkannt, wenn sich unten und/oder oben zu weit von der Mitte entfernen bzw. wenn unten und/oder oben zulasten der Mitte anwachsen (für den sozialwissenschaftlichen Diskurs siehe z. B. Heinze 2011; Mau 2012; Nachtwey 2016; Schöneck und Ritter 2018). Die sinnhafte Einordnung sozioökonomischer Unterschiede ist mithin eingebettet in eine semantische Architektur, in der die Mitte die (implizite) Referenzgröße für normative Urteile ob der (Un-)Gerechtigkeit sozialer Differenzen abgibt.

Vor dem Hintergrund dieser Ausgangsbeobachtung rekonstruiert der vorliegende Beitrag, wie im Zuge der Thematisierung des Zustands, der Befindlichkeiten und der Anliegen der (gesellschaftlichen) Mitte(lschicht) ${ }^{1}$ kollektive Vorstellungen von sozialer Ungleichheit konstituiert werden. Dabei wird von der Prämisse ausgegangen, dass die Kategorie der Mitte konstitutiv mit Ungleichheit verbunden ist, und zwar weil ihr - trotz aller Deutungsoffenheit und -bedürftigkeit - zwei miteinander verbundene semantische Gehalte strukturell eingeschrieben sind: Diskursive Praktiken, die mit der Mitte operieren, erzeugen erstens unweigerlich ein Außen, da es andernfalls keine Mitte geben würde, und zweitens bringen sie Innen und Außen in eine normative Ordnung, da die Mitte kulturgeschichtlich mit dem „Normalen“, dem „Geordneten“ und dem „Angemessenen“ verbunden ist (detailliert hierzu Abschn. 2). Hiervon ausgehend unternimmt der Beitrag eine interpretative Analyse von erzählerischen Praktiken, die auf die gesellschaftliche Mitte bzw. die Mittelschicht Bezug nehmen, um aus diesen Erzählungen Vorstellungen über die (Un-)Gerechtigkeit so-

\footnotetext{
1 Im vorliegenden Beitrag werden „Mittelschicht“ und „gesellschaftliche Mitte“ als Synonyme behandelt, obgleich sie strenggenommen unterschiedliche Bedeutungsgehalte tragen: Während die Kategorie der Mittelschicht eine vertikale Differenzierung impliziert, etabliert die Kategorie der gesellschaftlichen Mitte bzw. der Mitte der Gesellschaft eine konzentrische Anordnung.
} 
zioökonomischer Unterschiede zu destillieren. Grundlage der Analyse sind mehr als 2500 Zeitungsartikel, die zwischen 2005 und 2015 in der Süddeutschen Zeitung und der Frankfurter Allgemeinen Zeitung erschienen sind und Begrifflichkeiten aus der Wortfamilie der Mitte aufrufen, um soziopolitische Konstellationen zu beschreiben und zu beurteilen (vgl. Barbehön und Haus 2018; Barbehön und Geugjes 2019; Barbehön 2020; Barbehön et al. 2020).

Anhand dieser Analyse eröffnet der Beitrag einen doppelten Einblick in die Logik der Vermittlung von Ungleichheit: Zum einen werden die erzählerischen Praktiken erfasst, über die komplexe und bisweilen widersprüchliche gesellschaftliche Verhältnisse vermittelt im Sinne von greifbar und zugänglich gemacht werden, und zum anderen wird rekonstruiert, wie diese Verhältnisse über eine Vermittlung im Sinne einer Kopplung mit der Semantik der Mitte einer normativen Wertung zugeführt werden. Die Analyse liefert darüber hinaus einen Schlüssel zum Verständnis gegenwärtig wirksamer Rationalitäten des wohlfahrtsstaatlichen Regierens, insofern Erzählungen über die gesellschaftliche Mitte ein kollektives Wissen etabliert und reproduziert, was es bedeutet, „rational“ zu regieren (im Sinne von Foucault 2000).

\section{Bedeutungen der Mittelschicht}

„Mittelschicht“ ist ein bedeutungsoffener und deutungsbedürftiger Begriff. Die Sozialstrukturanalyse, die von Amts wegen mit der Definition, Operationalisierung und empirischen Identifikation der Mittelschicht betraut ist, wartet mit einer ganzen Reihe von Zugängen auf, die um den Titel der ,richtigen“ oder zumindest ,,plausiblen Messung" konkurrieren. Vom Einkommen über den Bildungsgrad und die berufliche Stellung bis hin zu Wertorientierungen und Formen der Lebensführung finden sich hier sowohl „harte“ als auch „weiche“ Indikatoren, die je nach Ausbuchstabierung $\mathrm{zu}$ je unterschiedlich großen und verfassten Mittelschichten führen (für einen Überblick siehe Atkinson und Brandolini 2013; Mau 2014). Neben dieser methodischtechnischen Kontroverse ist die Mittelschicht zugleich prominente Darstellerin gesellschaftsdiagnostischer Aufführungen. In der Beobachtung der modernen, kapitalistisch organisierten Gesellschaft und ihrer bisweilen scharfen sozioökonomischen Gegensätze dient die Bezugnahme auf die Mittelschicht seit jeher legitimatorischen Zwecken, insofern der Nachweis der Existenz einer großen und stabilen gesellschaftlichen Mitte gegen eine radikale Kapitalismuskritik und ihre Annahme einer notwendigen Zweiteilung der Gesellschaft in Bourgeoisie und Proletariat in Stellung gebracht werden kann. Vor diesem Hintergrund verwundert es nicht, dass der Status und die Zukunft der Mittelschicht in zeitdiagnostischen Beiträgen regelmäßig als Indikator für die Beurteilung des gesamtgesellschaftlichen Wohlbefindens dient (Beise 20092; Heinze 2011; Mau 2012; Nachtwey 2016); Beiträge, die für sozial-

\footnotetext{
2 Das Buch des Journalisten Marc Beise - Die Ausplünderung der Mittelschicht. Alternativen zur aktuellen Politik (2009) - ist in diesem Zusammenhang besonders eindrücklich, da es in das Verlagsprogramm der Bundeszentrale für politische Bildung und damit in den Kanon des gleichsam staatlich autorisierten Wissens über die Mittelschicht aufgenommen wurde.
} 
wissenschaftliche Verhältnisse häufig eine bemerkenswerte Resonanz auch in nichtwissenschaftlichen Kreisen finden.

Die Mittelschicht lebt mithin ein ambivalentes Leben. Einerseits scheint es keine Einigkeit dahingehend zu geben, was und wo sie genau ist, andererseits und zugleich wird in öffentlichen Debatten ständig auf sie rekurriert, oftmals ohne, dass präzisiert werden müsste, was der Begriff denn nun genau bezeichnen soll. Als Diskursgemeinschaft scheinen wir eine ungefähre Vorstellung davon zu haben, was mit dem Begriff gemeint ist und dass es um etwas gesamtgesellschaftlich Bedeutsames geht, wenn er aufgerufen wird. Diskurstheoretisch gesprochen lässt sich die Mittelschicht somit als „leerer Signifikant“ (vgl. Laclau 2005, S. 69ff.) interpretieren, d.h. als Produkt einer diskursiven Praxis, in der eine Vielzahl von differierenden und zum Teil widersprüchlichen Artikulationen durch ein vermeintlich Allgemeines überformt wird. So besehen stützen sich die notorische Unbestimmtheit und die diskursive Allgegenwärtigkeit der Kategorie der Mittelschicht wechselseitig ab (vgl. Barbehön et al. 2018): Sie kann deshalb eine derart prominente Stellung in Praktiken der gesellschaftlichen Selbstbeobachtung einnehmen, weil sie unbestimmt ist, heterogene Forderungen in sich aufnimmt und in enger Beziehung zum Gemeinwohl steht.

Die besondere semantische Strahlkraft, die von der Kategorie der Mittelschicht ausgeht und sie in die Nähe des Gemeinwohls rückt, lässt sich auf zwei Bedeutungsgehalte zurückführen, die der Semantik der Mitte eingeschrieben sind. Erstens ist die Mitte ein relationaler Begriff, der konstitutiv mit dem verbunden ist, was er nicht ist. Während diese Eigenschaft ein grundsätzliches Merkmal sprachlicher Zeichen ist, insofern gemäß (post-)strukturalistischem Denken Bedeutung aus der Differenz zwischen Zeichen entsteht (de Saussure 2001 [1931], S. 103ff.; Foucault 1973, S. 67ff.), weist der Begriff der Mitte bereits von sich aus auf sein außen hin: Mitte bezeichnet einen Zwischenraum, der erst dann entsteht, wenn dessen Begrenzungen mitgedacht werden. Eine Mitte ,an sich“ gibt es nicht; sie bringt notwendigerweise eine Skala oder einen Raum mit, auf der bzw. in dem sie eine Mittelstellung reklamiert. Zweitens, und auf Vorherigem aufbauend, ist die Mitte nicht einfach ein beliebiger Punkt auf einer Skala oder in einem Raum, sondern einer von besonderer Bedeutung. Das Außen, das eine Bezugnahme auf die Mitte zwangsläufig miterzeugt, wird in eine normative Ordnung gebracht: Die Mitte bezeichnet (in der westlichen Moderne) „das Normale“ oder „das Wünschenswerte“, wohingegen alles andere „,darunter“ oder „darüber“ bzw. ,am Rand“ oder „,im Extremen“ liegt. Diese semantischen Konnotationen lassen sich kulturgeschichtlich mindestens bis Aristoteles' Mesotes-Lehre zurückverfolgen, die das Tugendhafte als einen Punkt zwischen zwei Extremen lokalisiert (vgl. Münkler 2014; Barbehön et al. 2020, S. 19ff.). Ihren modernen Ausdruck findet dieses Prinzip etwa in der Wendung Mitte und Maß. Der Begriff der Mitte kann somit gar nicht gebraucht werden, ohne in das Fahrwasser von Wertigkeiten und Normativität zu geraten.

Im Falle des Begriffs der Mittelschicht folgt daraus, dass dessen Verwendung eine ganze Architektur gesellschaftlicher Ordnung etabliert. Er entwirft das Bild einer vertikal geschichteten Gesellschaft, die sich in ein „oben“, „unten“ und „dazwischen" aufteilt, wobei sich innerhalb der drei Schichten wiederum analoge Binnendifferenzierungen einziehen lassen (z.B. ,untere Mittelschicht“). Die Mittelschicht 
ist dabei existentiell von ihrem Außen abhängig: In einer (imaginativen) Gesellschaft, in der alle über die gleichen sozioökonomischen Ressourcen verfügen, wäre es widersinnig, von einer Mittelschicht zu sprechen, da oben und unten fehlen. Zugleich ist diesem Bild eine implizite Normativität eingeschrieben, insofern die Mittelschicht weder an dem einen noch an dem anderen Ende der Skala, sondern eben in der Mitte zwischen den Extremen sitzt. Die normative Anziehungskraft der gesellschaftlichen Mitte lässt sich nicht zuletzt an Umfragen zur Selbstverortung in einer vertikalen Hierarchie erkennen, bei denen sich die Befragten ganz überwiegend in der gesellschaftlichen Mitte eingruppieren (vgl. Mau 2012, S. 32), Friedrich Merz eingeschlossen. ${ }^{3}$ Auf symbolischer Ebene zeigt sich die Aufladung in den Metaphern des gesellschaftlichen „Kerns“ oder „Herzens“, die in der öffentlichen Debatte regelmäßig im Umfeld des Mittelschichtsbegriffs auftauchen (siehe hierzu die Materialanalyse in Abschn. 4).

Diese Bedeutungsgehalte, die dem Begriff der Mittelschicht strukturell inhärent sind bzw. die er sich kulturgeschichtlich angeeignet hat, sind von besonderer Relevanz für die Thematisierung sozialer Ungleichheit. Zunächst impliziert die Vorstellung einer geschichteten Gesellschaft die Existenz sozialer Unterschiede, die im Vergleich zu den Differenzen in einer Klassengesellschaft, wie sie etwa im angelsächsischen Raum mit dem Begriff der middle class aufgerufen wird, weniger stark ausgeprägt erscheinen und Bewegungen zwischen den Segmenten erlauben. Für die Frage, welches Ausmaß an sozialer Ungleichheit als vertretbar und welches als problematisch, welches als gerecht und welches als illegitim zu gelten hat, gibt die Vorstellung eines mittleren Segments die (implizite) Bezugsgröße ab, insofern nicht $\mathrm{zu}$ viele $\mathrm{zu}$ weit nach unten verlorengehen bzw. nach oben entfliehen dürfen, um nicht einen Zustand der „Polarisierung“ oder „Spaltung“ entstehen zu lassen und die „Schere“ weiter zu öffnen (siehe hierzu auch die Befunde zur Bedeutung der Mittelschicht in Ungleichheitsdiskursen bei Smith Ochoa 2020). In einer kapitalistisch organisierten Gesellschaft, in der (je nach Betrachtungsweise) die Existenz von oben und unten entweder unvermeidbar, gerechtfertigt oder wünschenswert ist, besetzt die Mitte den Raum des „Normalen“, da sie nicht einfach mehr als diese und weniger als jene hat, sondern weder zu viel noch zu wenig, also genau richtig viel.

Diese gesellschaftlichen Ordnungsvorstellungen sind schließlich eng verwoben mit Vorstellungen darüber, was wohlfahrtsstaatliche Politik zu leisten habe. Einer gängigen Position (die die Komplexität des Verhältnisses freilich radikal reduziert) zufolge stehen Oben, Mitte und Unten in einem jeweils spezifischen Verhältnis gegenüber wohlfahrtsstaatlicher Umverteilung: Während redistributive Maßnahmen für gewöhnlich als Vorteil für die „Unterschicht“ und als Nachteil für die „Oberschicht“ thematisiert werden, fällt die Bilanz für die ,Mittelschicht“, was auch immer mit diesem Begriff im Diskurs bezeichnet werden mag, ambivalent aus (Haus 2015, S. 153ff.). Es ist zumindest nicht unmittelbar offensichtlich, ob wohlfahrtsstaatliche Umverteilungen zugunsten oder zulasten der Mittelschicht ausfallen oder ob die

\footnotetext{
3 Der CDU-Politiker Friedrich Merz erklärte im Herbst 2018 in einem Interview, dass er sich (trotz eines Vermögens in Millionenhöhe) nicht der ,reichen Oberschicht“ zugehörig fühle, sondern der ,gehobenen Mittelschicht“; siehe https:/www.spiegel.de/wirtschaft/soziales/friedrich-merz-warum-er-nicht-zurmittelschicht-gehoert-a-1238635.html (aufgerufen am 27.11.2020).
} 
Mittelschicht in wohlfahrtsstaatlichen Arrangements an Autonomie gewinnt oder verliert, und somit können plausible argumentative Fäden sowohl in die eine als auch in die andere Richtung gesponnen werden. Das Verhältnis von Mittelschicht und Wohlfahrtsstaat ist uneindeutig, was aus erzähltheoretischer Perspektive einen Raum der Kontingenz eröffnet. So besehen wird verständlich, weshalb öffentliche Debatten, die mit der Mittelschichtskategorie hantieren, regelmäßig auf den Wohlfahrtsstaat zu sprechen kommen und die Rationalität seiner Regierungstechniken (im Sinne von Foucault 2000, 2005) im Verhältnis zur - selbst wiederum diskursiv konstruierten - Mittelschicht vermessen. ${ }^{4}$

Die bisherigen Ausführungen zu den Bedeutungsgehalten der Kategorie der Mitte(lschicht) sowie zum Verhältnis von Mittelschicht und Wohlfahrtsstaat sollen nicht den Anschein erwecken, die diskursive bzw. erzählerische Stellung der Mittelschicht sei strukturell determiniert. Vielmehr ist die Kategorie eingebettet in einen Bedeutungshorizont, der unterschiedliche Konfigurationen und Ausdeutungen ermöglicht (und andere unplausibel erscheinen lässt). Im Folgenden wird dargelegt, wie diesen Konfigurationen und Ausdeutungen material nachgegangen werden soll.

\section{Material und Auswertung}

Das diesem Beitrag zugrundeliegende Material entstammt einem Forschungsprojekt zum Zusammenhang von Mittelschichtsdiskursen und wohlfahrtsstaatlichem Regieren im Vergleich Deutschlands, Großbritanniens und Schwedens (vgl. Barbehön und Haus 2018; Barbehön und Geugjes 2019; Barbehön 2020; Barbehön et al. 2020). Das Projekt rekonstruierte aus einer interpretativen Perspektive heraus, wie ,die Mittelschicht“ zu einer bedeutungstragenden Entität und zum Gegenstand sozialpolitischer Steuerungsversuche wird. Der konzeptionelle Ausgangspunkt ist somit keine sozialstrukturelle Definition, sondern eine konstruktivistische Zugangsweise, die ,die Mittelschicht“ als bewegliches Produkt diskursiver Praktiken begreift (vgl. Wahrman 1995): Was ,die Mittelschicht“ ist, wer ihr zugehört, durch welche Eigenschaften sie sich auszeichnet, wo ihre Grenzen liegen, in welchem Zusammenhang sie mit dem gesamtgesellschaftlichen Wohlbefinden steht und welche Bedeutung ihr (deshalb) in unterschiedlichen Feldern wohlfahrtsstaatlicher Politikgestaltung zukommt, wird hiernach nicht durch eine vermeintlich objektive und eindeutig messbare sozialstrukturelle Position bestimmt, sondern durch die Art und Weise, wie über die Mittelschicht in öffentlichen Debatten gesprochen wird, d.h. wie der (im vorherigen Abschnitt dargelegte) Bedeutungshorizont der Kategorie der Mittelschicht symbolisch ausgefüllt wird.

Zur Rekonstruktion der Mittelschichtsdiskurse haben wir auf die Medienberichterstattung zurückgegriffen. In der modernen Gesellschaft können Massenmedien als der zentrale „Ort“ angesehen werden, an dem kollektives Wissen über soziale Verhältnisse etabliert und reproduziert wird (vgl. Fairclough 1995; Keppler 2005).

\footnotetext{
${ }^{4}$ Diese Überlegungen gelten insbesondere für den Typus des konservativen Wohlfahrtsstaates, für den die Absicherung von sozioökomischen Unterschieden, die auf unterschiedliche ,Leistungen auf dem freien Markt“ zurückgerechnet werden, ein wesentliches Leitmotiv darstellt (Esping-Andersen 1990, S. 26ff.).
} 
Dies gilt insbesondere für „die Mittelschicht“ (in ihrem Verhältnis zu Politik und Gesellschaft), die sinnlich nicht unmittelbar zugänglich ist - wir können sie weder sehen, hören, riechen noch fühlen - und die damit zwangsläufig qua symbolisch vermittelter Referenzen verfügbar gemacht werden muss. Die Massenmedien können hier als bevorzugter Ort gelten, um zu ermitteln, was eine Gesellschaft über sich selbst weiß (Luhmann 1996). Innerhalb des massenmedialen Raums haben wir auf Tageszeitungen zurückgegriffen, die sich für textanalytische Auswertungen besonders eignen. Im Falle Deutschlands haben wir das Material aus der Frankfurter Allgemeinen Zeitung sowie der Süddeutschen Zeitung als den zwei meistaufgelegten Zeitungen mit unterschiedlicher politischer Orientierung gewonnen. Zur Zusammenstellung des Korpus haben wir die Zeitungsarchive für die Jahre 2005 und 2015 mittels des Begriffs mitt* durchsucht und alle Artikel aufgenommen, in denen auf die gesellschaftliche, politische oder wirtschaftliche Mitte Bezug genommen wird. Für die dazwischenliegenden Jahre 2006 bis 2014 haben wir eine stärker fokussierte Materialerhebung ergänzt, aus der diejenigen Artikel hervorgegangen sind, in denen die Mittelschicht dezidiert mit dem Wohlfahrtsstaat in Beziehung gesetzt wird. Im Ergebnis resultierten daraus insgesamt 2668 Zeitungsartikel, die wir mittels der Software Maxqda einer interpretativen Analyse unterzogen haben. Ziel der Auswertung war es, die der Mittelschicht zugewiesenen Merkmale sowie deren Einbettung in politische Diagnosen, Forderungen und Argumente zu systematisieren. ${ }^{5}$

Für den vorliegenden Beitrag wurde das Material nochmals im Lichte der Kategorie des Narrativs gesichtet, die im ursprünglichen Projekt keine zentrale analytische Rolle einnahm (siehe aber Haus 2015). Narrative sollen hier verstanden werden als ,ein zentrales diskursstrukturierendes Regelsystem“ (Viehöver 2011, S. 194, Hervorh. entf.). Dies bedeutet zweierlei. Zum einen haben wir es bei Narrativen mit symbolischen Entitäten zu tun, die uns in Diskursen begegnen, wobei Diskurs, im poststrukturalistisch-archäologischen Sinne (Foucault 1973), die Gesamtheit an Aussageereignissen meint, die einem gemeinsamen Formationssystem zugehören. Im Verhältnis zu Diskursen liegen Narrative auf einer eigenen Ordnungsebene, insofern sie einzelne Aussageereignisse (Begriffe, Metaphern, Forderungen, Argumente etc.) zu einer Synthesis höherer Ordnung integrieren, indem sie bspw. Ursache-Wirkungsbeziehungen herstellen. Zum anderen wirken Narrative strukturierend bzw. reglementierend, da sie Aussageereignisse in vorgefertigte (indes niemals vollständig fixierte) Bahnen lenken. So können sich einzelne Forderungen oder Argumente anhand des etablierten Bestands an kollektiv verfügbaren Narrativen mit Relevanz, Plausibilität und Wirkmacht versorgen; oder weitreichender formuliert: sie müssen dies tun, um überhaupt Sinn zu ergeben. Insofern sind Narrative nicht allein symbolische Strukturphänomene, die sich in Diskursen finden, sondern sie wirken selbst strukturierend auf die Ereignishaftigkeit diskursiven Geschehens. Auf das vorliegende Material bezogen haben wir es hiernach mit einem bundesdeutschen

\footnotetext{
5 In einem weiteren empirischen Schritt haben wir leitfadengestützte Interviews mit politischen Entscheidungsträger*innen aus unterschiedlichen wohlfahrtsstaatlichen Politikfeldern geführt, um zu ermitteln, inwiefern das kollektive Wissen über „die Mittelschicht“ für die Entwicklung und Begründung sozialpolitischer Positionen von Bedeutung ist. Da diese Komponente des Forschungsprojekts für die Fragestellung des vorliegenden Beitrags nicht unmittelbar relevant ist, wird an dieser Stelle auf weitere Ausführungen verzichtet; für die Ergebnisse hierzu siehe Barbehön et al. (2020).
} 
Mittelschichtsdiskurs zu tun, der sich (unter anderem) aus verschiedenen Narrativen zusammensetzt.

Da das zur Verfügung stehende Material ursprünglich mit dem Ziel zusammengestellt wurde, die diskursive Konstruktion der Mittelschicht zu erschließen, konnten die im vorliegenden Beitrag interessierenden Aushandlungen sozioökonomischer Differenzen nicht auf gleichsam direktem Wege ermittelt werden. Für den vorliegenden Beitrag wurden daher im Zuge einer neuerlichen Materialanalyse Narrative rekonstruiert, in deren Zentrum die Mittelschicht steht, um diese Narrative sodann hinsichtlich der expliziten Thematisierungen von und impliziten Implikationen für soziale(r) Ungleichheit zu befragen. Da Narrative eine höhere Ordnungsebene besetzen, wurden die aus dem ursprünglichen Forschungsprojekt stammenden Materialcodierungen - die Definitionen und Abgrenzungen der Mittelschicht, der Rekurs auf die Mittelschicht in diagnostischen Aussagen über den Zustand der Gesellschaft sowie die Einbettung der Mittelschichtskategorie in politische Forderungen und Argumente - auf Clusterbildungen und innere Zusammenhänge hin untersucht, woraus sukzessive und in abstrahierenden Schritten Narrative konstruiert wurden. So zeigte sich etwa im Zuge der Materialsichtung, dass Aussagen, die Mittelschichtsmitglieder als gebildet, selbstverantwortlich und hart arbeitend ansprechen, bevorzugt im Umfeld der Begrifflichkeiten der Leistung und des Verdiensts auftauchen, vielfach mit der metaphorischen Beschreibung der Mittelschicht als „Herz“ oder „Rückgrat“ der Gesellschaft verbunden sind und typischerweise ein Bild vom Wohlfahrtsstaat zeichnen, dessen Aufgabe es ist, den durch Leistung erbrachten sozialen Status der Mittelschicht gegen Gefährdungen abzusichern. Aus diesen Einzelelementen ergibt sich das „Narrativ der verdienstvollen Mittelschicht“ (siehe Abschn. 4.2), das im Material selbst nie in Reinform auftritt und insofern eine Konstruktion der*des Forschenden darstellt (vgl. Münch 2016, S. 85 f.).

\section{Narrative Vermittlungen der Ungleichheit im deutschen Mittelschichtsdiskurs}

Im Zuge der Analyse des deutschen Mittelschichtsdiskurses konnten vier Narrative destilliert werden, die sich untereinander nochmals in eine Ordnung bringen lassen. Auf gleichsam grundlegendster Ebene findet sich das „Narrativ der Mittelschichtsgesellschaft" (Abschn. 4.1), das die Bundesrepublik als eine Gesellschaft präsentiert, die historisch und im internationalen Vergleich von einer starken Mittelschicht geprägt ist und die sich für den wirtschaftlichen Erfolg und die politische Stabilität des Landes maßgeblich verantwortlich zeichnet. Wir haben es hierbei mit einem Metanarrativ (vgl. Viehöver 2011, S. 200f.) zu tun, d.h. mit einer abstrakteren erzählerischen Konfiguration, die wesensähnlichen, aber eher kleinteiligen und gegenstandsbezogenen Narrativen einen Bedeutungshorizont zur Verfügung stellt. Ein Metanarrativ hilft zu verstehen, ,how the conflicting policy narratives on a certain issue can all be the case at the same time“" (van Eeten 2007, S. 256) - im vorliegenden Fall also, wie es sein kann, dass mit der Mittelschichtskategorie unterschiedliche Erzählungen entwickelt werden können, die bisweilen miteinander konkurrieren, aber gleichermaßen ,sinnvoll“" erscheinen. 
Eine Ebene unterhalb dieses Metanarrativs lassen sich drei weitere (sich bisweilen überlappende) Narrative identifizieren: das bereits angesprochene „Narrativ der verdienstvollen Mittelschicht“ (Abschn. 4.2), das „Narrativ der bedrohten Mittelschicht“ (Abschn. 4.3) sowie das „Narrativ der bevorzugten Mittelschicht“ (Abschn. 4.4). Sie alle beziehen ihre grundlegende Plausibilität aus der Imagination der deutschen Gesellschaft als einer um die Mitte zentrierten Gesellschaft, deuten diese Eigenschaft jedoch auf spezifische Art und Weise aus und produzieren damit je eigene Perspektiven auf die Gestalt, Ursachen und (Il-)Legitimität von sozioökonomischen Differenzen. Diese Narrative werden (vermutlich) von unterschiedlichen Kräften vorgetragen und sind somit immer auch Mittel des Kampfes um die Besetzung des leeren Signifikanten der Mitte. Eine solche akteurszentrierte Perspektive wird in der folgenden Analyse jedoch nicht eingenommen; vielmehr soll es darum gehen, die Erzählungen im Hinblick auf ihren Beitrag zur Etablierung einer kollektiv wirksamen symbolischen Ordnung von Ungleichheit zu interpretieren. Es wird mithin nicht danach gefragt, wer erzählt, sondern was und wie erzählt wird.

\subsection{Das Metanarrativ der Mittelschichtsgesellschaft}

Mediale Beschreibungen, worin die Wesenszüge der deutschen Gesellschaft bestehen, kommen immer wieder auf eine Formel zurück: „Das gesellschaftliche Leitbild der Mittelschichtgesellschaft“ (FAZ, 20.11.2005). Hiernach sei das wesentliche „Erfolgsmerkmal“" (SZ, 27.08.2015) der Bundesrepublik, nicht zuletzt im historischen Kontrast zur gesellschaftlichen Realität jenseits von Elbe und Oder, schon immer die Existenz einer breiten Mittelschicht gewesen, und auch heute noch könne man, zumindest im Vergleich mit anderen westlichen Industrienationen, auf ein starkes mittleres Segment in der Gesellschaftsstruktur blicken. Der historische Erfolg der jungen Bundesrepublik, die sich nach dem Zweiten Weltkrieg rasch stabilisiert und ein Wirtschaftswunder hervorgebracht habe, wird dabei auf die Existenz einer gefestigten und leistungsstarken Mittelschicht zurückgeführt, woraus ein grundlegender Zusammenhang extrapoliert wird: „In der Geschichte haben sich häufig diejenigen Gesellschaften als stabil erwiesen, die eine gesunde Mittelschicht haben. Wer in sich ruht und sich gerecht behandelt fühlt, rebelliert nicht, er sucht keine Feindbilder; auch das war bisher eine Stärke der deutschen Gesellschaft.“ (SZ, 19.06.2010)

In diesem Sinne sei die Mittelschicht „das Rückgrat der Gesellschaft“ (SZ, 31.05.2008), das „stabilisierende Scharnier in der Gesellschaft“ (SZ, 14.06.2008), „Träger [sic] von Demokratie und Fortschritt“ (FAZ, 10.01.2011) und ,moderierende Kraft“ (FAZ, 15.07.2008) - schlechthin ,der Kitt, der alles zusammenhält“ (SZ, 20.11.2006). Diese an Metaphern reichen Wendungen werden regelmäßig gekreuzt vom Begriff des Mittelstands als dem wirtschaftlichen Äquivalent zur Vorstellung einer um die Mitte zentrierten Gesellschaftsstruktur sowie von der Formel der sozialen Marktwirtschaft als einer (vermeintlichen) Harmonie zwischen Kapitalismus und sozialem Ausgleich (diskursanalytisch hierzu Nonhoff 2006), wobei sich bisweilen symbiotische Beziehungen einstellen: „Die Verheißung der Sozialen Marktwirtschaft, Wohlstand für alle zu schaffen, erfüllt sich nur in einer Mittelschichtgesellschaft“ (FAZ, 25.11.2006), und somit laufe die „Neubegründung der sozialen Marktwirtschaft [...] über die Neubegründung der Mittelschichten“ (SZ, 28.02.2005). 
Das Metanarrativ der Mittelschichtsgesellschaft kann dabei auf eine lange Historie zurückblicken, deren sozialwissenschaftliches Erbe eng mit der stilbildenden Beschreibung Deutschlands als einer ,nivellierten Mittelstandsgesellschaft“" (Schelsky 1953) verbunden ist; eine Formel, die auch in gegenwärtigen soziologischen Zeitdiagnosen als historische (Kontrast-)Folie eine wesentliche Rolle spielt (z. B. Reckwitz 2017, S. 273ff.; siehe hierzu auch Barbehön et al. 2018, S. 144ff.).

Im Hinblick auf die Ordnung von Ungleichheit produziert das Metanarrativ der Mittelschichtsgesellschaft eine Welt, in der fundamentale ökonomische Differenzen zunächst nicht vorgesehen sind, da sich die gesellschaftliche Wirklichkeit im Wesentlichen in der Mitte ereignet. Wer nicht Teil dieser Mitte ist, so die Erzählung, kann entweder durch Leistung (hierzu Abschn. 4.2) aufsteigen oder wird durch staatliche Interventionen eingefangen. Sozioökonomische Unterschiede, die auch in der sozialen Marktwirtschaft existieren, denn sonst wäre sie keine Marktwirtschaft, sind somit nicht von sich aus problematisch, sondern erst in Relation zur Mittelschicht, etwa wenn sie schrumpft, verunsichert ist (hierzu Abschn. 4.3) oder der Zugang zu ihr qua sozialem Aufstieg blockiert ist. Dies macht verständlich, weshalb mediale Aussagen über den Zustand, die Entwicklung oder die Befindlichkeiten der Mittelschicht eine besondere diskursive Resonanz erzeugen, verweisen derartige Aussagen doch unweigerlich auf das große Ganze: „Ausgerechnet die Mittelschicht. Wenig anderes steht für das deutsche Wirtschaftsmodell wie dieser Begriff." (SZ, 28.08.2015) In diesem Sinne fungiert das Imaginativ der Mittelschichtsgesellschaft als narratives Fundament, auf das gleichsam kleinteiligere bzw. stärker gegenstandsbezogene Narrative auf je spezifische Weise aufsatteln können, wie in den folgenden drei Abschnitten zu zeigen sein wird.

\subsection{Die verdienstvolle Mittelschicht}

Das Narrativ der verdienstvollen Mittelschicht konstruiert eine gesellschaftliche Mitte, in der sich diejenigen aufhalten, die sich ihren sozioökonomischen Status hart erarbeitet haben und deshalb berechtigterweise dort sind, wo sie sind - und das bedeutet auch: über anderen. Der Wohlfahrtsstaat wird dabei als eine ambivalente Einrichtung wahrgenommen. Einerseits wird ihm die Aufgabe zugeschrieben, die Verdienste und den Status der Mittelschicht abzusichern, und insofern erscheint er als Grundvoraussetzung einer stabilen Mitte. Andererseits wird der Wohlfahrtsstaat in seiner Abhängigkeit von den finanziellen Zuwendungen der Mittelschicht thematisiert, womit er stets in Verdacht steht, die Mittelschicht zu schröpfen.

Den metanarrativen Anknüpfungspunkt findet diese Erzählung im Topos des Aufstiegsversprechens, das der mittelschichtigen sozialen Marktwirtschaft eingeschrieben sei: „Wenn sich einer richtig anstrengt in der Ausbildung und danach im Beruf, kann er sich mehr leisten“ (SZ, 28.08.2015). Diese im Mediendiskurs apodiktisch vorgetragene Annahme korrespondiert mit der allgegenwärtigen Beschreibung der Mittelschicht als gebildet, hart arbeitend, autonom und verantwortungstragend. Mittelschichtsmitglieder zeichneten sich durch die Maxime aus, „das eigene Leben in die Hand zu nehmen, mit allen Risiken und Unwägbarkeiten“ (FAZ, 29.12.2007); Mittelschicht, das seien „Leute, die weit mehr verdienen als der Durchschnitt der 
Bevölkerung ${ }^{6}$, dafür aber auch hart und lange arbeiten - und viel Steuern zahlen“ (SZ, 09.03.2009). Dabei werden Bildung und Arbeit nicht nur als Mittel zum Zweck dargestellt, sondern sie treten auf im Gewand des „Leistungscredo[s]“ (SZ, 18.07.2012) oder der „bürgerliche[n] Arbeitsethik“ (SZ, 02.09.2006) und bezeichnen somit ,übergeordnete Werte“ (FAZ, 30.11.2011), die gerade von Mitgliedern der Mittelschicht anerkannt und in der eigenen Lebensführung beherzigt würden. Hinzu treten Leistungen nicht-monetärer Art: „Die Mitte der Gesellschaft, die sich ordentlich um ihre Kinder kümmere“ (FAZ, 18.06.2007) und ganz generell „,solidarisch“ (FAZ, 18.02.2010) sei.

Diese Klassifizierungen sind insofern bemerkenswert, als sie - notwendigerweise (siehe Abschn. 2) - implizieren, dass jenseits der Mittelschicht keine Bildung anzutreffen sei, nicht hart gearbeitet würde, keine Solidarität herrsche und die Kinder verkommen würden. Für die symbolische Ordnung von Ungleichheit hat dies weitreichende Konsequenzen, da relative Vorteile der Mittelschicht gegenüber den (unbenannten) anderen als grundsätzlich legitim erscheinen. Die Tatsache, dass einige auf der Sonnenseite der Ungleichheit stehen, hängt hiernach mit der individuellen Leistungsbereitschaft zusammen, von der diejenigen, die sich nicht in der Mitte aufhalten, offenbar zu wenig besitzen. Das Narrativ der verdienstvollen Mittelschicht ist somit nur auf den ersten Blick unverdächtig; auf den zweiten Blick offenbart es sich als Spiegelbild eines abwertenden Unterschichtsdiskurses, der all denjenigen, die nicht als Mittelschichtsmitglieder identifiziert werden, Defizite und Versäumnisse zurechnet (vgl. Lessenich 2009, S. 267 f.; Schram 2012, S. 242). So hat etwa Kettenring (2017, S. 58ff.) gezeigt, dass der Diskurs um die „,neue Unterschicht“ (z. B. Nolte 2005) eine soziale Gruppe entwirft, die mit ihrer mangelnden Leistungsbereitschaft die sich ihr bietenden Aufstiegschancen schlichtweg nicht ergreifen würde; sie verhalte sich nicht wie die Mittelschicht es tut, und deshalb sei ihre sozioökonomische Stellung legitim, und nicht etwa Ausdruck eines gesamtgesellschaftlichen Problems. Das Narrativ der verdienstvollen Mittelschicht ist an der Produktion dieser Deutung (wenn auch unfreiwillig) beteiligt, und zwar aufgrund der symbolischen Logik der Mittelschichtskategorie auch dann, wenn es die „Unterschicht“ gar nicht benennt.

Für diesen soziale Ungleichheiten absichernden Effekt ist der Begriff der Leistung von zentraler Bedeutung. Die Mittelschicht arbeite nicht nur, um ihren Lebensunterhalt zu sichern, sondern sie leiste etwas, d.h. sie trägt zur Funktionsfähigkeit eines größeren Ganzen bei. Im medialen Diskurs werden die Mitglieder der Mittelschicht regelmäßig als „Leistungsträger“ (z.B. SZ, 06.08.2005) oder als „leistungsbereit“ (z.B. FAZ, 05.09.2009) adressiert, als eine Gruppe, die ,die Bundesrepublik immer getragen [hat]“ (SZ, 12.12.2015), und insofern war „der Erfolg der Republik [...] der Erfolg der Mittelschicht“ (SZ, 07.05.2008). Kurzum: Die Mittelschicht sei „das unentbehrliche Rückgrat einer freien Gesellschaft [...], die ihren Wohlstand aus dem Leistungswillen, aus den Leistungsanreizen für jeden Einzelnen bezieht“" (SZ, 11.09.2006). Von dieser meritokratischen Deutungsfigur ist es dann nicht mehr weit zu Referenzen auf „Leistungsgerechtigkeit“ (z. B. SZ, 17.08.2010; FAZ, 06.07.2015)

\footnotetext{
${ }^{6}$ Eine verblüffende Formulierung, wird die Mittelschicht doch vielfach als mittlere Einkommensgruppe definiert.
} 
bzw. subtiler zur Idee einer „wohlverstandene[n] Gleichheit“ (SZ, 11.09.2006), die keine absolute Gleichheit meint, sondern eine (vermeintliche) Gleichheit hinsichtlich der Möglichkeiten, Leistung zu erbringen. ${ }^{7}$ Im Zusammenhang mit der Leistungssemantik ist zudem auffallend, dass die zweite Bewegungsrichtung des meritokratischen Prinzips - der Abstieg aus der Mitte im Falle abnehmender Leistungserbringung - offenbar nicht vorgesehen ist (vgl. Barbehön et al. 2020, S. 179). Ein Schrumpfen der Mittelschicht (hierzu Abschn. 4.3) wird in aller Regel nicht als „gerechte“ Konsequenz unterschiedlicher Performanz erzählt, sondern als Ausdruck systemischer Dysfunktionalität, womit bereits an dieser Stelle das meritokratische Ideal zu wanken beginnt.

Für den Wohlfahrtsstaat ergibt sich aus dieser Erzählung ein Balanceakt. Einerseits erscheint er fundamental abhängig von der ,leistungsbereite[n] Mittelschicht, die $94 \%$ der Einkommensteuern“ (SZ, 07.01.2009) zahle, und andererseits wird immer wieder eine durch Steuern und Abgaben ,geschröpfte Mitte der Gesellschaft“ (FAZ, 20.05.2008) bzw. ,schwer gebeutelte Mittelschicht“ (FAZ, 10.08.2010) beklagt. Das Narrativ der verdienstvollen Mittelschicht zeichnet ein ambivalentes, bisweilen widersprüchliches Bild der gesellschaftlichen Mitte, die zugleich als leistungsbereit und -fähig, als autonom und selbstverantwortlich imaginiert wird, nur um an anderer Stelle als bedroht und geradezu zerbrechlich zu erscheinen, wenn zu viel ihrer Leistungen staatlich abgeschöpft werden. Dahinter liegt eine - verzerrte oder mindestens stark vereinfachte (vgl. Goodin und Le Grand 1987; Hilpert 2012) - Vorstellung von sozialstaatlicher Umverteilung, wonach der Wohlfahrtsstaat aus der Mitte nimmt und nach unten weitergibt. Vor dem Hintergrund der narrativen Konstruktion der Mittelschicht als der Leistungsträgerin muss dieser Weg geradezu problematisch erscheinen, hat die „Nichtmittelschicht“ doch kein verdienstvolles Verhalten an den Tag gelegt.

\subsection{Die bedrohte Mittelschicht}

Die zuletzt genannte Deutung, wonach die Mittelschicht latent gefährdet ist, steht im Zentrum des Narrativs der bedrohten Mittelschicht. Im Unterschied zur Verdiensterzählung tritt die Mittelschicht hier nicht primär als leistungsstarke und autonome Gruppe auf, die hier und da zu stark belastet wird, sondern als fundamental verunsichert und als Prekariat von morgen (Haus 2015, S. 160ff.). Im Zentrum des Narrativs stehen die Metaphern der Schere, der Kluft oder des Abschmelzens, die eine dramatische Schrumpfung bildlich greifbar machen. Die Mittelschicht ist hier weniger agierende Kraft denn passives Objekt, das sich anonymen Kräften wie der Globalisierung ausgesetzt sieht. Unter diesen Bedingungen mögen einige wenige den Aufstieg nach oben schaffen, die meisten jedoch taumeln am Abgrund, wenn

\footnotetext{
7 Diese mediale Thematisierung korrespondiert mit den Ergebnissen der Studien von Laenen et al. (2019) und Heuer et al. (2020), in denen Vorstellungen über Meritokratie, Verdienst und Bedürftigkeit anhand von Fokusgruppengesprächen ermittelt wurden. Smith Ochoa (2020, S. 331) weist in diesem Zusammenhang darauf hin, dass das Deutungsmuster der Meritokratie einen diskursiven Knotenpunkt darstellt, über den unterschiedliche Erzählungen über Ungleichheit zueinander in Beziehung treten.
} 
sie nicht bereits abgerutscht sind (siehe hierzu auch die ,storyline of decline“ bei Smith Ochoa 2020, S. 329ff.).

Während die Mittelschicht in der Verdiensterzählung zumeist als Kollektivsubjekt ohne Außenwelt auftritt, wird sie in der Bedrohungserzählung stets in Beziehung zu oben und unten gesetzt. Es geht dann um ,diese - schrumpfende - Mitte zwischen Arm und Reich“ (SZ, 14.04.2009), um die Sorge und Nöte einer ,immer stärker abschmelzenden Mittelschicht“ (SZ, 29.12.2012) oder um die „Vergrößerung der Ober- und der Unterschicht zu Lasten der Mittelschicht“ (FAZ, 15.07.2008). Vor dem Hintergrund des Metanarrativs der Mittelschichtsgesellschaft sind diese Tendenzen nicht nur, und womöglich nicht einmal primär, ein Problem für die Mittelschicht selbst, sondern vor allem für die Gesellschaft insgesamt, die sich ,,in Richtung einer Gesellschaft ohne Mittelstand und einer Polarisierung zwischen oben und unten" bewege, was wiederum ,nicht dem gesellschaftlichen Frieden dient“" (SZ, 01.02.2005). Apodiktisch formuliert: „Die Gesellschaft gerät aus dem Gleichgewicht. Normale [!] Menschen müssen hohe Risiken auf sich nehmen, um sich durchzuschlagen. Die Mittelschicht gerät unter Druck.“ (FAZ, 02.07.2015) In diesem Sinne sei die Tatsache, dass „die arbeitende Mittelschicht kleiner wird, [...] ein Signal“ (SZ, 10.05.2008), und zwar ein Signal an alle, dass etwas mit den sozioökonomischen Unterschieden nicht stimmt, sind doch zunehmend „normale Menschen“ die Leidtragenden.

Die metanarrative Fundierung, die der Erzählung der bedrohten Mittelschicht ihre Plausibilität und Sprengkraft verleiht, wird bisweilen explizit gemacht, und zwar durch historische Abgleiche. Die Zeit, in denen Schelsky noch von der nivellierten Mittelstandsgesellschaft sprach, ,,ist vorbei. Deutschland ist eine Klassengesellschaft - wenn auch eine ohne Bewusstheit für die sowohl feineren wie auch deutlich gröberen Unterschiede“ (SZ, 09.02.2005). Die nivellierte Mittelstandsgesellschaft - die übrigens von Schelsky (1953, S. 231) nicht als Ausdruck sozialen Ausgleichs gedeutet, sondern mit „Auflösung und Dekomposition“ in Verbindung gebracht wurde, aber darüber gehen die medialen Debatten (wie auch einige sozialwissenschaftliche Kommentator*innen) in der Erinnerung an Schelsky großzügig hinweg (siehe Barbehön et al. 2018, S. 144ff.) - sei „,verblichen“, und stattdessen „eine neue gesellschaftliche Dreiteilung entstanden, die zwar nichts mit den alten Klassen zu tun hat, aber sehr viel mit neuen Bedingungen einer globalisierten Wirtschaft" (SZ, 11.09.2006). Diese Zeit, in der ,die Mitte dem Staat etwas wert“ und ,das stabilisierende Scharnier in der Gesellschaft [war]", sei, womöglich unwiederbringlich, vorbei - ,,in der Mitte wird es einsam“ (SZ, 14.06.2008). Dabei gilt erneut: Die Thematisierung sozioökonomischer Unterschiede erhält in dem Moment eine alarmierende Note, wo sie nicht einfach ,nur“ wachsende soziale Differenzen feststellt, sondern die Mittelschicht, und mit ihr das Normale des gesellschaftlichen Ganzen, betroffen sieht. Eine Gefährdung der Mittelschicht sei schlechthin ,,gesellschaftszerstörend“, weil damit „das Bindeglied zwischen unten und oben“ verlorenginge (FAZ, 20.11.2006).

Im Narrativ der bedrohten Mittelschicht nehmen sozialstrukturelles Wissen und das Deutsche Institut für Wirtschaftsforschung (DIW) als einer seiner wesentlichen Konstrukteure eine besondere Stellung ein. Die Diagnose einer fundamentalen Bedrohung der gesellschaftlichen Mitte ist eng verknüpft mit der statistischen Dauerbeobachtung der Bevölkerung. Neben der schwierigen Frage danach, wie die Mit- 
telschicht mittels quantitativer Indikatoren gefasst werden kann, ist im vorliegenden Zusammenhang vor allem interessant, wie der mediale Diskurs diachrone Veränderungen statistischer Kennzahlen in kollektive Gefühlslagen transformiert. Die Abnahme der Größe der mittleren Einkommensgruppen, die typischerweise unter dem Begriff der „Schrumpfung“ und damit als tendenziell dramatische Entwicklung auftritt, wird regelmäßig übersetzt in eine „Statuspanik“ (SZ, 15.06.2010), in „ökonomische Abstiegsängste“ (FAZ, 15.07.2005), in „Statusverlustängste“ (FAZ, 18.07.2008), in ,soziale Absturzängste“ (FAZ, 31.07.2005), in ,eine Anhäufung von Unzufriedenheit, durchwirkt von Existenzangst“ (SZ, 02.01.2007), in eine Schädigung der „souveräne[n] Selbstsicherheit“ (SZ, 07.05.2008). Die „German Angst“ (FAZ, 22.01.2015) macht gerade auch vor der Mittelschicht nicht halt, womit sich die Sorge um die bedrohte Mittelschicht zu einer ganzen Gefühlswelt auswächst.

Diese narrative Mixtur aus „objektiver“ Statusbedrohung und ,subjektivem“ Gefühlschaos hat profunde Auswirkungen darauf, was als angemessener Umgang mit sozioökonomischer Ungleichheit erscheint. Da das gesamtgesellschaftliche Wohlbefinden maßgeblich vom Status der Mittelschicht abzuhängen scheint, erhält eine Politik für die Mitte (was immer das im Einzelnen bedeuten mag) den Anstrich einer Politik für alle und damit auch für die weniger gut Situierten: „Es wird klar, dass es auch dem unteren Drittel nur dann besser gehen kann, wenn das mittlere Drittel prosperiert“ (SZ, 02.09.2006). Den bedrohten und verunsicherten Mitgliedern der Mittelschicht, „den Leistungsträgern des Alltags“, müsse die Angst vor dem sozialen Abstieg genommen werden, um somit ,die Gesellschaft zu stabilisieren und neue Chancen auch für das untere Drittel der Gesellschaft zu schaffen“ (SZ, 31.08.2006). Zum anderen impliziert die Erzählung von der gleichermaßen verunsicherten wie systemrelevanten Mittelschicht eine gleichsam entschuldigende Haltung gegenüber jenen mittelschichtigen Praktiken, die in Verdacht stehen, soziale Ungleichheiten zu reproduzieren oder zu verschärfen. Ein augenscheinliches Beispiel hierfür ist die Thematisierung des Bildungssystems, das sich zunehmend zur „Kampfzone zwischen den Milieus“ entwickle: „Die ,bildungsfernen“ Eltern fordern mehr Hilfe, die traditionellen Bildungseliten mehr Auslese“ (FAZ, 25.01.2015). Dadurch sei ein „Wettrüsten der Elternhäuser“ (SZ, 04.12.2013) entstanden, das zu einer Verfestigung sozialer Differenzen führe - die „Frage dabei ist: Überziehen es die Eltern inzwischen? Und noch eine Befürchtung gibt es: Bleiben die Kinder der Unterschicht auf der Strecke?"“(FAZ, 20.09.2015) Während sich das Narrativ der bedrohten Mittelschicht somit auch zu einer Kritik mittelschichtiger Verhaltensweisen zuspitzen kann, wird diese Kritik sogleich durch die Tatsache abgeschwächt, dass die Mittelschicht als Opfer von Prekarisierung erscheint - und wer soll es ihr da verübeln, wenn sie sich, und damit die Gesellschaft als Ganze, zu retten versucht, auch wenn das bisweilen zulasten anderer geschieht?

\subsection{Die bevorzugte Mittelschicht}

Die zuletzt angesprochenen kritischen Klänge ertönen am lautesten im Narrativ der bevorzugten Mittelschicht. Diese Erzählung steigert das Metanarrativ der Mittelschichtsgesellschaft zur Diagnose eines strukturellen Mitte-Bias, der zu einer überbordenden Bedeutung der Mittelschicht in Politik und Gesellschaft geführt habe. Da 
die Mitte der bevorzugte Raum im politischen Wettbewerb sei (vgl. Link 1991) und sich Mittelschichtsmitglieder aufgrund ihres sozialen und kulturellen Kapitals besonders artikulationsfähig präsentierten, habe sich ein System der wohlfahrtsstaatlichen „Verhätschelung“ (Haus 2015, S. 163ff.) etabliert, das allenfalls gegen Widerstand und mit hohen politischen Kosten aufgebrochen werden könne. Die Mittelschicht profitiere somit über ihre berechtigten Ansprüche hinaus vom Status quo, womit die „wirklich Bedürftigen“ zuweilen aus dem Blick gerieten.

Das Narrativ der bevorzugten Mittelschicht tritt zumeist in Gestalt einer schleichenden historischen Entwicklung auf. Die „fetten Jahre“ hätten aus der Bundesrepublik „einen Gewerkschafts- und Sozialstaat für die Mittelschicht gemacht“ (SZ, 18.08.2005), der unter den gegenwärtigen wirtschaftlichen Bedingungen zunehmend an seine Grenzen stoße. Da „Wahlen in der Mitte gewonnen werden“ (SZ, 20.06.2005), habe sich die politische Logik, „die finanzielle Mittelschicht $\mathrm{zu}$ umwerben und die finanzielle Unterschicht noch weiter abgleiten zu lassen“ (SZ, 02.04.2009), über Zeit fest etabliert. So seien etwa die gesetzliche Rentenversicherung eine „Veranstaltung für die Mittelschichten“ (SZ, 08.04.2005), das Ehegattensplitting im Steuerrecht eine „Subvention“ für die Mittelschicht (SZ, 20.10.2015) oder das Elterngeld eine „Wohltat für das Mittelschichtenpublikum“ (FAZ, 23.04.2008). Und da die ,wahlentscheidende Mittelschicht“ (FAZ, 13.11.2005) auch künftig „die Regeln des politischen Spiels“ bestimmen würde, „wird der deutsche Sozialstaat bleiben, was er seit langem ist: eine Umverteilungsmaschine von der Mittelschicht an die Mittelschicht“"(SZ, 02.06.2006). Die vom Metanarrativ der Mittelschichtsgesellschaft bereitgestellte Deutung tritt hier somit in Form einer Systemlogik auf, die gleichsam hinter den Rücken der politischen Akteure wirke und geradezu zwangsläufig dazu führe, dass eine wohlfahrtsstaatliche Unwucht zugunsten der gesellschaftlichen Mitte entstanden ist.

Noch verstärkt werde diese Systemlogik durch eine aktive Rolle der Mittelschicht, die wisse, wie man sich Gehör verschafft und sich durchsetzt. „Für die Mittelschicht, welche die öffentliche Debatte bestimmt" (SZ, 30.01.2010), sei es strukturell einfacher, sichtbar in Erscheinung zu treten; es seien die Mitglieder der Mittelschicht, „die in Deutschland reden dürfen, weil sie über den Status und die Techniken verfügen, die dazu nötig sind“ (FAZ, 15.03.2015). Im Falle politischer Maßnahmen, die nicht ihren Interessen entsprächen, könne die Kritik der Mittelschicht ,gewaltig werden“ (SZ, 08.07.2008), was dazu führe, ,dass die Politik sich an den Aktiven und Vernehmbaren orientiert, während die Passiven und Stillen übergangen werden“ (FAZ, 09.11.2015). Gemäß der Erzählung der bevorzugten Mittelschicht ist die Mittelschicht somit nicht zuletzt deshalb bevorzugt, weil sie weiß, wie sie eine Bevorzugung erreicht und verteidigt.

Im Unterschied zum Bedrohungs- und Verunsicherungsnarrativ, in dem mittelschichtige Abgrenzungspraktiken (vgl. Schöneck und Ritter 2018), zumindest hier und da, ebenfalls einem kritischen Blick zugeführt werden, agiert die Mittelschicht im Bevorzugungsnarrativ von einer Position der Stärke aus und auf den eigenen Vorteil bedacht, ,gepaart mit dem Wunsch nach Besitzstandswahrung“ (SZ, 09.05.2008). Einerseits wird sie also mitverantwortlich gemacht für sozioökonomische Ungleichheiten, da sie „Anschluss zur Oberschicht“ suche und sich gleichzeitig „nach unten“ abgrenze (FAZ, 20.09.2015). Andererseits jedoch tritt die Erzählung 
nicht als diametraler Gegensatz zum Metanarrativ der Mittelschichtsgesellschaft auf, sie problematisiert also etwa nicht die Deutung, die Mitte sei das „Herz“ und der „leistungsfähige Motor“ der Gesellschaft. Trotz seines kritischen Gehalts muss auch das Narrativ der bevorzugten Mittelschicht als Ausdruck des Metanarrativs bzw. als dessen Kehrseite gedeutet werden, da es nur als Korrektiv auftritt, sobald die Mittelschicht den Bogen zu überspannen scheint. Die anhand der obigen Erzählungen rekonstruierte symbolische Ordnung von Ungleichheit wird somit nicht grundsätzlich infrage gestellt, sondern „,nur“ gedeckelt bzw. eingehegt.

Auffallend ist zudem, dass sich das Narrativ der bevorzugten Mittelschicht in erster Linie um finanzielle Vorteile dreht. Nicht die Annahme eines besonderen Fleißes, einer hohen Leistungsfähigkeit und generell einer normativen Höherwertigkeit der Mittelschicht gegenüber ,den anderen“ sind hier Zielscheibe von Problematisierungen, sondern die monetären Zuwendungen, die hier und da zu hoch ausfielen. Auch im Falle eines kritischen Blicks steht die Mittelschicht somit für eine überlegene Lebensweise, womit wiederum das Grundmuster der symbolischen Ordnung von Differenzen reproduziert wird. Dies stellt einen auffälligen Kontrast gegenüber dem britischen Mittelschichtsdiskurs dar, in dem die „middle class“ als eine unter mehreren soziokulturellen Lebenswelten erscheint, die etwa im Verhältnis zur ,working class“ nicht in einem Verhältnis der Hierarchie steht (vgl. Barbehön et al. 2020, S. 138ff.).

\section{Schluss}

Ungleichheit, so der Ausgangspunkt dieses Beitrags, muss vermittelt werden, und zwar in zweifacher Hinsicht: einerseits ist sie als gesellschaftliches Makrophänomen sinnlich nicht direkt erfahrbar, sondern muss über symbolische Praktiken (wie etwa die der Erzählung) in die Sphäre der kollektiven Wahrnehmung übermittelt werden; und sie ist andererseits gebunden an die Imagination einer gesellschaftlichen Mitte, mit deren Hilfe die Grenze zwischen sozialen Unterschieden und sozialer Ungleichheiten gezogen wird. Eine Analyse der narrativen Konstruktionen der Mittelschicht bzw. der gesellschaftlichen Mitte erlaubt es daher, die in einer Gesellschaft kursierenden Verständnisse über (un)gewöhnliche, (un)problematische und (il)legitime Erscheinungsformen sozioökonomischer Differenzen zu entschlüsseln. Gegenüber einem direkten Zugriff auf Ungleichheitserzählungen (hierzu jüngst Smith Ochoa 2020) hat dieser Zugang den Vorteil, auch den impliziten Wissensbeständen über Ungleichheit, die den Thematisierungen des „Normalen“ eingeschrieben sind, auf die Spur kommen zu können.

Für die deutsche mediale Öffentlichkeit konnten anhand einer interpretativen Analyse drei Narrative rekonstruiert werden, die auf je spezifische Weise an der Konstruktion einer Ungleichheitsordnung beteiligt sind: das Narrativ der verdienstvollen Mittelschicht, das die Mitte als Ort der Autonomie und des Fleißes konstruiert, sodass soziale Differenzen solange unproblematisch sind, wie sie als Ausdruck von Leistung erscheinen; das Narrativ der bedrohten Mittelschicht, das sich um den sozialen Abstieg aus der Mitte sorgt, da es hieran eine gesamtgesellschaftlich bedeutsame Verschärfung sozialer Ungleichheit abliest; schließlich das Narrativ der 
bevorzugten Mittelschicht, das einen kritischen Blick auf die Bevorteilung der Mittelschicht zulasten der ,wirklich Bedürftigen“ wirft (dabei aber die Annahme einer Höherwertigkeit mittelschichtiger Lebensführung unangetastet lässt). Alle drei Narrative bewegen sich innerhalb des symbolischen Raums des Metanarrativs der Mittelschichtsgesellschaft, das die Bundesrepublik als ein traditionell um die Mitte zentriertes Gemeinwesen beschreibt, in dem aufsteigen kann, wer wirklich aufsteigen will. Das Metanarrativ bildet die Klammer für die drei zuvor genannten Narrative, die sich im Fahrwasser der Imagination Deutschlands als einer Mittelschichtsgesellschaft mit Plausibilität versorgen und damit den Topos zugleich reproduzieren.

Diese narrativen Entwürfe der Mittelschicht sind dabei nicht nur für die Konstruktion einer Ungleichheitsordnung von Relevanz, sondern auch für die Rationalität wohlfahrtsstaatlichen Regierens. Die Gleichzeitigkeit von Allgegenwärtigkeit und normativer Aufladung macht die Kategorie der Mittelschicht zu einem wesentlichen Bezugspunkt politischer Kontroversen, und zwar insbesondere in sozialpolitischen Feldern, in denen es um die Bearbeitung von Ungleichheit geht. Aus den oben rekonstruierten Narrativen ergeht der Auftrag an den (konservativen) Wohlfahrtsstaat, auch und gerade über die gesellschaftliche Mitte zu wachen, ihre Leistungen abzusichern und sie vor sozialem Abstieg zu schützen, ohne freilich die ,wirklich Bedürftigen“ zu vergessen - doch da auch sie von einer starken Mitte profitieren, kommt die Sorge um die Mittelschicht letztlich einer Sorge um das Gemeinwesen gleich. Der Mittelschichtsdiskurs schränkt somit systematisch ein, welche Orientierungen und Prinzipien als politisch angemessen und funktional erforderlich gelten (was einen erzähltheoretischen Zugang insbesondere für die Analyse wohlfahrtsstaatlicher Reformen sinnvoll erscheinen lässt; vgl. Blum und Kuhlmann 2019).

Am plakativsten tritt dieser Effekt an der Tatsache hervor, dass die in politischen Kontroversen häufig artikulierte Forderung, es bedürfe einer „Politik für die Mittelschicht“, vielfach ohne weitere Begründungsleistungen auskommt. Dass eine Politik für die Mitte nie verkehrt sein kann und letztlich zum Wohle aller ist, scheint derart selbstevident zu sein, dass allein der apodiktische Ausspruch politische Resonanz erzeugt. Es handelt sich, so Foucault (2005, S. 1014) in der Beschreibung von ,,politischer Rationalität“, um Evidenz, ,die wir für gesichert halten“. Zugleich ist aufgrund der Deutungsoffenheit der Kategorie der Mittelschicht hochgradig variabel, was eine Politik für die Mitte konkret bedeutet, womit sich politische Forderungen als Versuch beschreiben lassen, den leeren Signifikanten der Mitte zu besetzen (Analoges geschieht im Parteienwettbewerb, wenn von allen und jedem die Repräsentation der ,politischen Mitte“ reklamiert wird). Die diskursive Kategorie der Mittelschicht und die Praktiken ihrer narrativen Verarbeitung können hinsichtlich ihrer politischen Wirkmächtigkeit mithin kaum überschätzt werden.

Danksagung Mein Dank gilt Sonja Blum und Johanna Kuhlmann für ihre wertvollen Hinweise zu einer früheren Fassung dieses Beitrags sowie Marilena Geugjes und Michael Haus für die gemeinsame Projektarbeit, in der die hier angestellten Überlegungen ihren Ursprung haben.

Funding Open Access funding enabled and organized by Projekt DEAL.

Open Access Dieser Artikel wird unter der Creative Commons Namensnennung 4.0 International Lizenz veröffentlicht, welche die Nutzung, Vervielfältigung, Bearbeitung, Verbreitung und Wiedergabe in jeglichem Medium und Format erlaubt, sofern Sie den/die ursprünglichen Autor(en) und die Quelle ord- 
nungsgemäß nennen, einen Link zur Creative Commons Lizenz beifügen und angeben, ob Änderungen vorgenommen wurden.

Die in diesem Artikel enthaltenen Bilder und sonstiges Drittmaterial unterliegen ebenfalls der genannten Creative Commons Lizenz, sofern sich aus der Abbildungslegende nichts anderes ergibt. Sofern das betreffende Material nicht unter der genannten Creative Commons Lizenz steht und die betreffende Handlung nicht nach gesetzlichen Vorschriften erlaubt ist, ist für die oben aufgeführten Weiterverwendungen des Materials die Einwilligung des jeweiligen Rechteinhabers einzuholen.

Weitere Details zur Lizenz entnehmen Sie bitte der Lizenzinformation auf http://creativecommons.org/ licenses/by/4.0/deed.de.

\section{Literatur}

\section{Verwendete Literatur}

Atkinson, Anthony B., und Andrea Brandolini. 2013. On the identification of the middle class. In Income inequality: economic disparities and the middle class in affluent countries, Hrsg. Janet C. Gornick, Markus Jäntti, 77-100. Stanford: Stanford University Press.

Barbehön, Marlon. 2020. Reclaiming constructivism: towards an interpretive reading of the "Social Construction Framework". Policy Sciences 53(1):139-160.

Barbehön, Marlon, und Marilena Geugjes. 2019. Caught in the middle? Welfare state legitimisation and problematisation in German and Swedish middle-class discourse. International Journal of Politics, Culture, and Society 32(1):1-20.

Barbehön, Marlon, und Michael Haus. 2018. How central is the middle? Middle class discourses and social policy design in Germany. In Creating target publics for welfare policies: a comparative and multilevel approach, Hrsg. Lorenzo Barrault-Stella, Pierre-Edouard Weill, 49-66. New York, London: Springer.

Barbehön, Marlon, Marilena Geugjes, und Michael Haus. 2018. Separate, but central?: Distinktionspraktiken und Normalitätsanspruch der Mittelschicht in der medialen Repräsentation. In Die Mitte als Kampfzone: Wertorientierungen und Abgrenzungspraktiken der Mittelschichten, Hrsg. Nadine M. Schöneck, Sabine Ritter, 143-159. Bielefeld: transcript.

Barbehön, Marlon, Marilena Geugjes, und Michael Haus. 2020. Middle class and welfare state. Making sense of an ambivalent relationship. London, New York: Routledge.

Beise, Marc. 2009. Die Ausplünderung der Mittelschicht. Alternativen zur aktuellen Politik. Bonn: Bundeszentrale für politische Bildung.

Blum, Sonja, und Johanna Kuhlmann. 2019. Stories of how to give or take-towards a typology of social policy reform narratives. Policy and Society 38(3):339-355.

Burzan, Nicole. 2011. Soziale Ungleichheit. Eine Einführung in die zentralen Theorien, 4. Aufl., Wiesbaden: VS.

van Eeten, Michel J.G. 2007. Narrative policy analysis. In Handbook of public policy analysis: theory, politics, and methods, Hrsg. Frank Fischer, Gerald J. Miller, und Mara S. Sidney, 251-269. Boca Raton, London, New York: CRC Press.

Esping-Andersen, Gøsta. 1990. The three worlds of welfare capitalism. Cambridge: Polity Press.

Fairclough, Norman. 1995. Media discourse. London: Edward Arnold.

Foucault, Michel. 1973. Archäologie des Wissens. Frankfurt am Main: Suhrkamp.

Foucault, Michel. 2000. Die „Gouvernementalität“. In Gouvernementalität der Gegenwart: Studien zur Ökonomisierung des Sozialen, Hrsg. Ulrich Bröckling, Susanne Krasmann, und Thomas Lemke, 41-67. Frankfurt am Main: Suhrkamp.

Foucault, Michel. 2005. Die politische Technologie der Individuen. In Schriften in vier Bänden. Dits et Ecrits: Band IV: 1980-1988, 999-1015. Frankfurt am Main: Suhrkamp.

Goodin, Robert E., und Julian Le Grand. 1987. Not only the poor. The middle classes and the welfare state. London: Allen and Unwin.

Haus, Michael. 2015. Mittelschicht und Wohlfahrtsstaat - Drei Deutungsmuster und ihre Relevanz für die Zukunft eines wohlfahrtsstaatlichen Grundkonsenses. Zeitschrift für Sozialreform 61(2):147-170.

Heinze, Rolf G. 2011. Die erschöpfte Mitte. Zwischen marktbestimmten Soziallagen, politischer Stagnation und der Chance auf Gestaltung. Weinheim: Juventa. 
Heuer, Jan-Ocko, Thomas Lux, Steffen Mau, und Katharina Zimmermann. 2020. Legitimizing inequality: the moral repertoires of meritocracy in four countries. Comparative Sociology 19(4-5):542-584.

Hilpert, Dagmar. 2012. Wohlfahrtsstaat der Mittelschichten? Sozialpolitik und gesellschaftlicher Wandel in der Bundesrepublik Deutschland (1949-1975). Göttingen: Vandenhoeck \& Ruprecht.

Keppler, Angela. 2005. Medien und soziale Wirklichkeit. In Mediensoziologie: Grundfragen und Forschungsfelder, Hrsg. Michael Jäckel, 91-106. Wiesbaden: VS.

Kettenring, Sara. 2017. Wer konstruiert die „Neue Unterschicht“? Eine Untersuchung der Bedeutung der Mittelschicht für die diskursive Verhandlung der Legitimität sozialer Ungleichheit. Masterarbeit am Institut für Politische Wissenschaft. Heidelberg: Universität Heidelberg.

Laclau, Ernesto. 2005. On populist reason. London, New York: Verso.

Laenen, Tijs, Federica Rossetti, und Wim van Oorschot. 2019. Why deservingness theory needs qualitative research: Comparing focus group discussions on social welfare in three welfare regimes. International Journal of Comparative Sociology 60(3):190-216.

Lessenich, Stephan. 2009. „Neue Mitte“: Das Ende der Planwirtschaft. In Prekarität, Abstieg, Ausgrenzung: Die soziale Frage am Beginn des 21. Jahrhunderts, Hrsg. Robert Castel, Klaus Dörre, 259-268. Frankfurt am Main: Campus.

Link, Jürgen. 1991. Links/rechts, Mitte/Extreme - Metamorphosen einer politischen Landschaft. Kulturrevolution: Zeitschrift für angewandte Diskurstheorie 26:19-24.

Luhmann, Niklas. 1996. Die Realität der Massenmedien. Opladen: Westdeutscher Verlag.

Mau, Steffen. 2012. Lebenschancen. Wohin driftet die Mittelschicht? Berlin: Suhrkamp.

Mau, Steffen. 2014. Die Mittelschicht - das unbekannte Wesen? Aus Politik und Zeitgeschichte 64(49):3-10.

Münch, Sybille. 2016. Interpretative Policy-Analyse. Eine Einführung. Wiesbaden: Springer VS.

Münkler, Herfried. 2014. Die Entstehung des Mitte-Paradigmas in Politik und Gesellschaft. Aus Politik und Zeitgeschichte 64(49):49-54.

Nachtwey, Oliver. 2016. Die Abstiegsgesellschaft. Über das Aufbegehren in der regressiven Moderne. Frankfurt am Main: Suhrkamp.

Nolte, Paul. 2005. Generation Reform. Jenseits der blockierten Republik. München: C.H. Beck.

Nonhoff, Martin. 2006. Politischer Diskurs und Hegemonie. Das Projekt „Soziale Marktwirtschaft“. Bielefeld: transcript.

Reckwitz, Andreas. 2017. Die Gesellschaft der Singularitäten. Zum Strukturwandel der Moderne. Berlin: Suhrkamp.

de Saussure, Ferdinand. 2001. Grundfragen der allgemeinen Sprachwissenschaft. Berlin: Walter de Gruyter.

Schelsky, Helmut. 1953. Wandlungen der deutschen Familie in der Gegenwart. Dortmund: Ardey.

Schöneck, Nadine M., und Sabine Ritter (Hrsg.). 2018. Die Mitte als Kampfzone. Wertorientierungen und Abgrenzungspraktiken der Mittelschichten. Bielefeld: transcript.

Schram, Sanford F. 2012. The deep semiotic structure of deservingness: discourse and identity in welfare policy. In The argumentative turn revisited: public policy as communicative practice, Hrsg. Frank Fischer, Herbert Gottweis, 236-268. Durham, London: Duke University Press.

Smith Ochoa, Christopher. 2020. Trivializing inequality by narrating facts: a discourse analysis of contending storylines in Germany. Critical Policy Studies 14(3):319-338.

Viehöver, Willy. 2011. Diskurse als Narrationen. In Theorien und Methoden, 3. Aufl., Bd. 1, Hrsg. Reiner Keller, Andreas Hirseland, Werner Schneider, und Willy Viehöver, 193-224. Wiesbaden: VS.

Wahrman, Dror. 1995. Imagining the Middle Class. The political representation of class in Britain, $c$. 1780-1840. Cambridge, New York: Cambridge University Press.

\section{Zitierte Zeitungsartikel}

FAZ, 15.07.2005. Eins im Sinn. Frankfurter Allgemeine Zeitung, 15.07.2005, Nr. 162, S. 31.

FAZ, 31.07.2005. Unsere Kanzlerin. Frankfurter Allgemeine Sonntagszeitung, 31.07.2005, Nr. 30, S. 19.

FAZ, 13.11.2005. Der Jungstar schweigt. Frankfurter Allgemeine Sonntagszeitung, 13.11.2005, Nr. 45, S. 11.

FAZ, 20.11.2005. Auf dünnem Eis. Frankfurter Allgemeine Sonntagszeitung, 20.11.2005, Nr. 46, S. 6.

FAZ, 20.11.2006. Berliner Einheit in Lafontaines Gegenwind. Frankfurter Allgemeine Zeitung, 20.11.2006, Nr. 270, S. 4.

FAZ, 25.11.2006. Auf der Suche nach dem Leitbild. Frankfurter Allgemeine Zeitung, 25.11.2006, Nr. 275, S. 12.

FAZ, 18.06.2007. Gutscheine statt Bargeld. Frankfurter Allgemeine Zeitung, 18.06.2007, Nr. 138, S. 4. 
FAZ, 29.12.2007. Zur Freiheit gehört Ungleichheit. Frankfurter Allgemeine Zeitung, 29.12.2007, Nr. 302, S. 3.

FAZ, 23.04.2008. Getriebene der Bundespolitik. Frankfurter Allgemeine Zeitung, 23.04.2008, Nr. 95, S. 3. FAZ, 20.05.2008. Die arme Mitte. Frankfurter Allgemeine Zeitung, 20.05.2008, Nr. 116, S. 1.

FAZ, 15.07.2008. Das Bewusstsein der Mittelschicht. Frankfurter Allgemeine Zeitung, 15.07.2008, Nr. 163, S. 8 .

FAZ, 18.07.2008. Mehr Lehrer und bessere Hauptschulen. Frankfurter Allgemeine Zeitung, 18.07.2008, Nr. 166, S. 10.

FAZ, 05.09.2009. Westerwelle umwirbt Mittelschicht. Frankfurter Allgemeine Zeitung, 05.09.2009, Nr. 206, S. 6.

FAZ, 18.02.2010. Darum geht es der FDP. Frankfurter Allgemeine Zeitung, 18.02.2010, Nr. 41, S. 10.

FAZ, 10.08.2010. Die SPD will auch die „Rente mit 67“ abräumen. Frankfurter Allgemeine Zeitung, 10.08.2010, Nr. 183, S. 2.

FAZ, 10.01.2011. Den Fortschritt neu entdecken. Frankfurter Allgemeine Zeitung, 10.01.2011, Nr. 7, S. 8. FAZ, 30.11.2011. „Wir sind pessimistischer“. Frankfurter Allgemeine Zeitung, 30.11.2011, Nr. 279, S. 6.

FAZ, 22.01.2015. Mittelstand verliert die Zuversicht. Frankfurter Allgemeine Zeitung, 22.01.2015, Nr. 18, S. 19.

FAZ, 25.01.2015. Im Zweifel sind die Lehrer schuld. Frankfurter Allgemeine Sonntagszeitung, 25.01.2015, Nr. 4, S. 6.

FAZ, 15.03.2015. Die Angst in den Augen der Frauen. Frankfurter Allgemeine Sonntagszeitung, 15.03.2015, Nr. 11, S. 37.

FAZ, 02.07.2015. Noch erscheinen die Diktatoren des Internets milde. Frankfurter Allgemeine Zeitung, 02.07.2015, Nr. 150, S. 13 .

FAZ, 06.07.2015. Die Starken stärken. Frankfurter Allgemeine Zeitung, 06.07.2015, Nr. 153, S. 8.

FAZ, 20.09.2015. Nachhilfe für das Einser-Abitur. Frankfurter Allgemeine Sonntagszeitung, 20.09.2015, Nr. 38, S. 23.

FAZ, 09.11.2015. Demokratie? Mehr oder weniger. Frankfurter Allgemeine Zeitung, 09.11.2015, Nr. 260, S. 8 .

SZ, 01.02.2005. Polarisierung zwischen oben und unten. Süddeutsche Zeitung, 01.02.2005, Nr. 25, S. 10.

SZ, 09.02.2005. Das neue Subproletariat. Süddeutsche Zeitung, 09.02.2005, Nr. 32, S. 11.

SZ, 28.02.2005. Mittelschicht muss gestärkt werden. Süddeutsche Zeitung, 28.02.2005, Nr. 48, S. 2.

SZ, 08.04.2005. Raus aus der Nein-Sager-Ecke. Süddeutsche Zeitung, 08.04.2005, Nr. 80, S. 20.

SZ, 20.06.2005. Wieder ist von zwei Spanien die Rede. Süddeutsche Zeitung, 20.06.2005, Nr. 139, S. 4.

SZ, 06.08.2005. „Das Spiel ist noch lange nicht aus“. Süddeutsche Zeitung, 06.08.2005, Nr. 180, S. 7.

SZ, 18.08.2005. Arbeitslose gegen Arbeitsplatzbesitzer. Süddeutsche Zeitung, 18.08.2005, Nr. 189, S. 11. SZ, 02.06.2006. Gespaltene Generation. Süddeutsche Zeitung, 02.06.2006, Nr. 126, S. 4.

SZ, 31.08.2006. Beck will Mitte der Gesellschaft für SPD gewinnen. Süddeutsche Zeitung, 31.08.2006, Nr. 200, S. 6.

SZ, 02.09.2006. Die bedrängte Mitte. Süddeutsche Zeitung, 02.09.2006, Nr. 202, S. 4.

SZ, 11.09.2006. Die Mitte der Gesellschaft - vom Staat geschröpft. Süddeutsche Zeitung, 11.09.2006, Nr. 209, S. 2.

SZ, 20.11.2006. Zorn, Angst und Hartz. Süddeutsche Zeitung, 20.11.2006, Nr. 267, S. 4.

SZ, 02.01.2007. Die Reform der Reformpolitik. Süddeutsche Zeitung, 02.01.2007, Nr. 1, S. 4.

SZ, 07.05.2008. Das Sichere ist nicht mehr sicher. Süddeutsche Zeitung, 07.05.2008, Nr. 106, S. 6.

SZ, 09.05.2008. Falsche Mittel gegen Angst. Süddeutsche Zeitung, 09.05.2008, Nr. 108, S. 4.

SZ, 10.05.2008. „Von Vertuschung kann keine Rede sein”. Süddeutsche Zeitung, 10.05.2008, Nr. 109, S. 6.

SZ, 31.05.2008. „Nächstenliebe ist keine staatliche Dienstleistung”. Süddeutsche Zeitung, 31.05.2008, Nr. 125, S. 6.

SZ, 14.06.2008. Und was wird aus uns? Süddeutsche Zeitung, 14.06.2008, Nr. 137, S. 1.

SZ, 08.07.2008. Eine Zauberformel für die Kita. Süddeutsche Zeitung, 08.07.2008, Nr. 157, S. 6.

SZ, 07.01.2009. Westerwelle vermeidet Koalitionsaussage. Süddeutsche Zeitung, 07.01.2009, Nr. 4, S. 6.

SZ, 09.03.2009. Die Attraktivität der FDP. Süddeutsche Zeitung, 09.03.2009, Nr. 56, S. 4.

SZ, 02.04.2009. Antworten auf die Fragen der Woche. Süddeutsche Zeitung, 02.04.2009, Nr. 77, S. 39.

SZ, 14.04.2009. Reichensteuer als Alibi. Süddeutsche Zeitung, 14.04.2009, Nr. 85, S. 4.

SZ, 30.01.2010. Was uns zusteht. Süddeutsche Zeitung, 30.01.2010, Nr. 24, S. 3.

SZ, 15.06.2010. Panik in der Mittelschicht. Süddeutsche Zeitung, 15.06.2010, Nr. 134, S. 6.

SZ, 19.06.2010. Brüder, zur Mitte. Süddeutsche Zeitung, 19.06.2010, Nr. 138, S. 4.

SZ, 17.08.2010. Am schönsten ist, wenn der Schmerz nachlässt. Süddeutsche Zeitung, 17.08.2010, Nr. 188, S. 2.

SZ, 18.07.2012. Die Mittelschicht in der Krise. Süddeutsche Zeitung, 18.07.2012, Nr. 164, S. 2. 
SZ, 29.12.2012. Mehr als Arm und Reich. Süddeutsche Zeitung, 29.12.2012, Nr. 300, S. 4.

SZ, 04.12.2013. Bitte nicht stören. Süddeutsche Zeitung, 04.12.2013, Nr. 280, S. 4.

SZ, 27.08.2015. Die Mittelschicht schrumpft. Süddeutsche Zeitung, 27.08.2015, Nr. 196, S. 1.

SZ, 28.08.2015. Die Tücken des Booms. Süddeutsche Zeitung, 28.08.2015, Nr. 197, S. 4.

SZ, 20.10.2015. Das Kalkül der Ehe. Süddeutsche Zeitung, 20.10.2015, Nr. 241, S. 4.

SZ, 12.12.2015. Schwarz-Rot-Grob. Süddeutsche Zeitung, 12.12.2015, Nr. 287, S. 13. 